\title{
Uric Acid and Plasma Lipids in Cerebrovascular Disease.* Part I, Prevalence of Hyperuricaemia
}

\author{
JOHN PEARCE, $\dagger$ M.D., M.R.C.P. ; HASAN AZIZ, $\ddagger$ M.B., B.SC., M.R.C.P.ED.
}

\begin{abstract}
Summary : Serum uric acid levels measured by the phosphotungstic sodium cyanide method have been estimated in 60 patients suffering from a cerebrovascular incident. Patients with diseases known to predispose to hyperuricaemia were excluded. Twenty-five per cent. of patients with acute strokes had hyperuricaemia, and this did not appear to be a transitory phenomenon. These findings suggest that hyperuricaemia may be one of the factors in the pathogenesis of atheroma.
\end{abstract}

"La goutte est aux artères ce que le rhumatisme est aux cœurs" (Huchard, 1899).

\section{Introduction}

The high incidence of atherosclerosis in patients suffering from gout has been known since the last century (Futcher, Osler, and McCrae, 1907). The increased incidence of hyperuricaemia in patients with coronary heart disease has been shown by Gertler, Garn, and Levine (1951), Kohn and Prozan (1959), and Dreyfuss (1960). More recently Breckenridge (1966) showed a significant relationship between hyperuricaemia and hypertension. Despite these important findings, little attention has been paid to the possible relation between hyperuricaemia and atherosclerotic and hypertensive disease of the cerebral vascular tree. Hansen (1965) found significant hyperuricaemia in $36 \%$ of 115 patients with acute cerebral infarction. Meyer, Kypros, and Gilroy (1964) found hyperuricaemia in $32 \%$ of 59 patients suffering from cerebrovascular disease.

A wider range of normal values has recently been established by epidemiological studies (Kellgren, 1964), and further investigation seemed indicated to validate the possible association of raised plasma uric acid levels and cerebrovascular disease.

\section{Material and Methods}

An unselected series of 60 consecutive patients with "strokes" admitted to the Combined Neurological Service and to the general medical wards at the Hull Royal Infirmary were investigated. Most of these patients were referred to the hospital as emergencies for an acute completed cerebrovascular episode, while some were admitted for investigations of recurrent transient cerebral ischaemic attacks.

The diagnosis of cerebral vascular disease was based on a detailed clinical examination, supplemented by contrast radiological studies in 19 selected patients (32\%). Conditions such as hyperthyroidism, hypothyroidism, polycythaemia, and other blood dyscrasias and dysproteinaemias were excluded, since these are known to alter uric acid levels. The presence of hypertension (diastolic pressure greater than $100 \mathrm{~mm}$. $\mathrm{Hg}$ ), diabetes mellitus, and antihypertensive treatment was specially noted. In addition to routine laboratory tests, measurements of serum uric acid, blood urea, and serum creatinine were carried out in

\footnotetext{
* Based on a paper read to Leeds and Newcastle Regional Physicians Meeting on 17 May 1969.

† Consultant Neurologist.

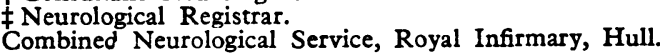

each patient. Patients with acute renal failure were not included in this study, and those with moderate or advanced renal disease were excluded. Transient azotaemia precipitated by dehydration, and subsequently reversed, accounts for the blood urea levels between 70 and $120 \mathrm{mg} . / 100 \mathrm{ml}$. in two patients (see Fig. 3). Blood samples were taken in the morning while the patients were fasting.

Serum uric acid was determined by the phosphotungstic sodium cyanide method (Pearson, Sterm, and Mackett, 1953), with measurements on an absorptiometer which gives a direct reading and eliminates the optical error apt to occur with other methods. Estimates were made on admission in all cases; serial estimates were recorded in 16 patients, the second value being obtained from three to nine months from the ictus. A control series of patients, matched for age and sex, was included for purposes of comparison of uric acid values from the same geographical area and performed in the same laboratory.

\section{Results}

Two hundred control subjects were examined for serum uric acid levels ; gout, treatment with diuretics or hypotensive drugs, thyroid disorders, severe hypertension, dysproteinaemias, and renal failure were excluded. The mean age of the 124 men was 54 years, and of the 76 women 66 years. These subjects are comparable to the series of patients with cerebrovascular disease.

Statistical analysis of the controls shows no trend for uric acid levels in respect of age or sex, though values in males are slightly higher, as in other series: Males: mean serum uric acid $=5 \cdot 28 \mathrm{mg} . / 100 \mathrm{ml}$., S.D. $=0.66$; mean +2 S.D.s $=6 \cdot 60$. Females: mean serum uric acid $=4.47 \mathrm{mg} . / 100 \mathrm{ml}$. , S.D. $=$ 0.78 ; mean +2 S.D.s $=6.03$.

In this study values of $7 \mathrm{mg} . / 100 \mathrm{ml}$. in males and $6 \mathrm{mg}$./ $100 \mathrm{ml}$. in females are therefore regarded as abnormal. These correspond to the values of Henry (1960) and Kellgren (1964).

The study included 57 patients $(95 \%)$ with cerebral infarction and $3(5 \%)$ with cerebral haemorrhage. There were 41 men with a mean age of 55 and 19 women with a mean age of 68. The different ages in each sex reflect the general trend for strokes to occur at older ages in women.

Fig. 1 shows that nine men had serum uric levels of $7 \mathrm{mg}$./ $100 \mathrm{ml}$. or more, an incidence of $22 \%$, and that six women had uric acid levels of $6 \mathrm{mg} . / 100 \mathrm{ml}$. or more, an incidence of $33 \%$. Fig. 1 also compares the uric acid levels with age in both men and women, and does not indicate any definite progressive rise of uric acid levels with increasing age.

Fig. 2 shows uric acid levels in association with diastolic blood pressure. There is no definite trend towards hyperuricaemia with high diastolic pressure in either sex.

Since patients with moderate or advanced renal failure were deliberately excluded, a valid comparison of uric acid levels with blood urea (see Fig. 3) was not possible. Nevertheless, of six patients with blood urea levels between 45 and $120 \mathrm{mg} . /$ $100 \mathrm{ml}$., four had hyperuricaemia. Similarly no valid comparison with serum creatinine levels (see Fig. 4) was possible in view of the small number of patients with creatinine levels greater than $1.7 \mathrm{mg} . / 100 \mathrm{ml}$. The exclusion of patients with 
gross renal failure was deliberate, and was designed to eliminate hyperuricaemia secondary to this factor, which might otherwise contribute to a falsely high incidence in this series.

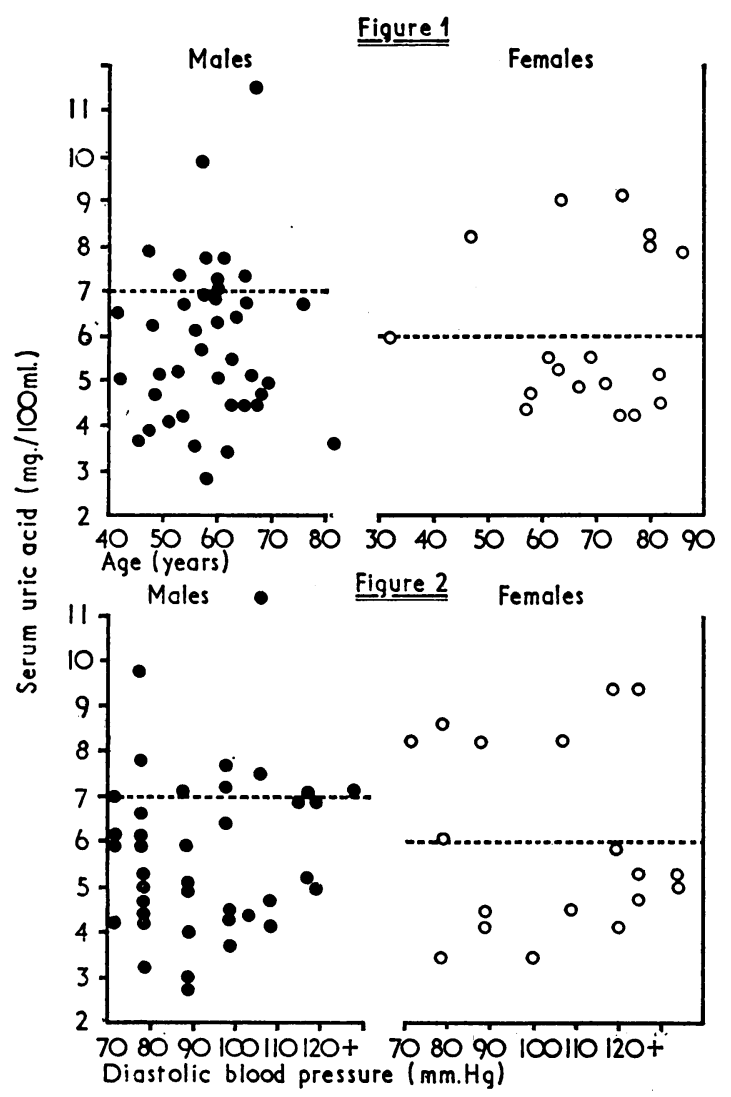

FIG. 1.-Serum uric acid levels related to sex and age. FIG. 2.- Serum uric acid levels related to diastolic pressure.

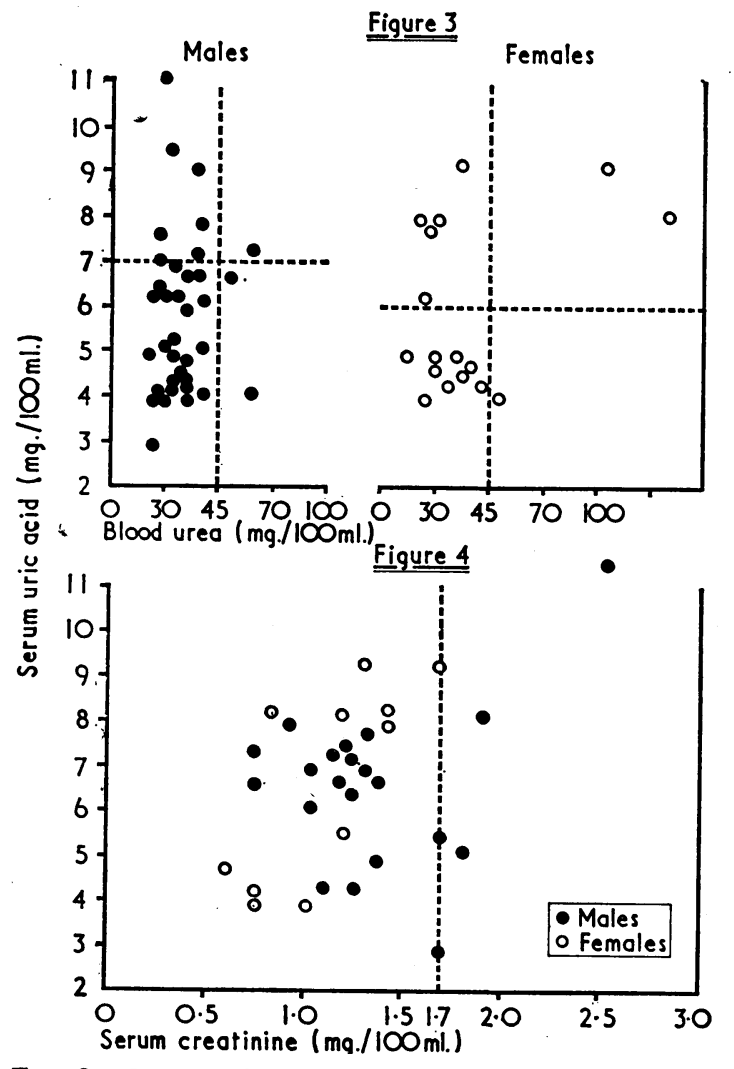

Fig. 3.-Serum uric acid levels related to blood urea. FIG. 4.-Serum uric acid levels related to serum
Fig. 5 shows the levels of serum uric acid in relation to the duration of the illness after the onset of the stroke. There is no indication that raised levels occur immediately after the acute illness and subsequently subside. Serial uric acid estimations were performed in 16 patients in whom the initial estimate had been made within two weeks of the onset. The results are shown in Fig. 6. No significant pattern of change in uric acid levels was found $(P \gg 0 \cdot 25)$.

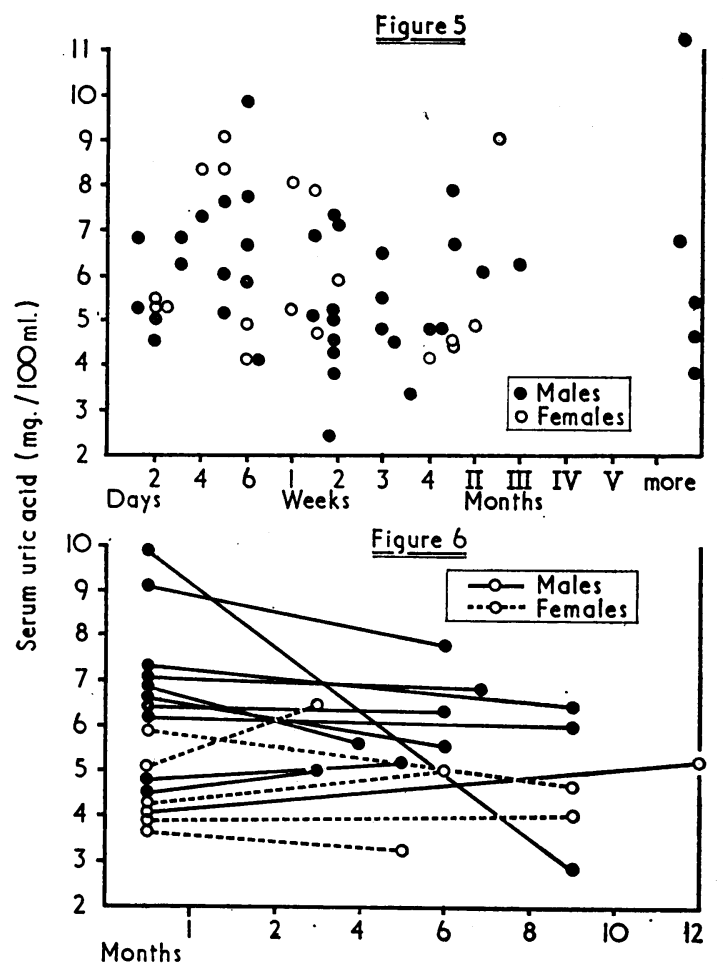

FIG. 5.-Serum uric acid levels related to duration of stroke. FIG. 6.-Serum uric acid levels, serial measurements after stroke.

\section{Discussion}

Reference has already been made to the association of raised uric acid levels in gout, coronary artery disease, and hypertension. Hyperuricaemia has also been demonstrated after the administration of diuretics and ganglion blocking drugs (Dollery, Duncan, and Schumer, 1960). Reports of raised serum uric acid levels in myxoedema, toxaemia of pregnancy, diabetes, psoriasis, multiple myeloma, renal failure, and mongolism have been summarized by Meyer et al. (1964).

The normal values of serum uric acid levels quoted in the literature have varied according to the method employed. Further, variations occur in relation to the total calorie intake and to the fat and the purine content of the diet, and there is a diurnal variation (Henry, 1960 ; Kellgren, 1964).

The results of the present study show that, despite wide normal variations for serum uric acid, a significant proportion of patients with cerebral infarction or cerebral haemorrhage show hyperuricaemia. This was found in $25 \%$ of patients in this study, and affects both sexes. These results are similar to those of Hansen (1965) and Meyer et al. (1964), who found hyperuricaemia in respectively $36 \%$ and $32 \%$ of their patients with cerebrovascular disease. Older and less accurate methods for estimating uric acid were used in these studies. From our own series patients with significant clinical renal failure were deliberately excluded. In patients with normal serum creatinine and blood urea levels, and in those with only slight rises in these measurements of renal function, there was no significant correlation between mild azotaemia and raised uric acid levels. 
Similarly, hypertension did not in itself predispose to hyperuricaemia in our series, but it is probable (Breckenridge, 1966) that sustained severe hypertension may be an important factor; this was not, however, apparent in the present study.

It seemed possible that raised uric acid levels might be a transitory phenoménon after a cerebral infarct or haemorrhage, but examination of this hypothesis excluded it as an explanation for the hyperuricaemia (Fig. 5). Further, serial estimations after a stroke showed only occasional and rather wide variation, but no distinctive pattern emerged.

It seems, therefore, that primary hyperuricaemia, in the absence of gout or any other known predisposing cause, is present in a significant proportion of patients with cerebral infarction or haemorrhage. This suggests that hyperuricaemia may be one of many factors predisposing to the genesis of atheroma and its sequelae in the cerebral vasculature. This suggestion correlates with the recent views that the renal lesion in gout is a "vascular nephrosclerosis" (Barlow and Beilin, 1968).

There is no satisfactory explanation at present of the role of uric acid in the causation of atherosclerosis. Traut, Knight, and Szanto (1954) demonstrated the presence of urate crystals in the proliferated intima of coronary arteries and in organized thrombi. Since atheroma is a generalized process, this suggests one possible mechanism by which urate deposits might be the starting-point for progressive atherosclerotic disease. The other explanation was put forward by Gertler et al. (1951). They suggested that uric acid, being a powerful cationic surface agent, could perhaps attach itself to the largar cholesterol molecule and bring it into contact with the arterial intima. In this way they envisaged the onset of atheroma. This idea was further elaborated by Schrade, Boehle, and Biegl:: (1960), who thought that atherosclerosis might be a "complex disturbance of biood lipid and other regulatory mechanisms," and ther felt that one of the factors involved was an abnormality in uric ac: metabol- ism. Apart from these speculations, there is no evidence from experimental pathology to suggest a mechanism which might relate abnormal uric acid levels to atheroma. One possible mechanism might be via abnormalities of plasma lipids, and therefore a further study has been carried out which forms the subject of a separate report (Pearce and Aziz, 1969).

We are indebted to Mr. J. Parks and his colleagues for the biochemical measurements of uric acid. Mr. E. Miller provided invaluable statistical advice.

\section{REFERENCES}

Barlow, K. A., and Beilin, L. J. (1968). Quarterly fournal of Medicine, 37, 79.

Breckenridze, A. (1966). Lancet, 1, 15.

Doller: C. T. Duncan, H., and Schumer, B. (1960), British Medical ournal, $2,832$.

Dreyfuss, F. (1960). Diseases of the Chest, 38, 332.

Futcher, T. B., Osler, W., and McCrae, T. (1907). Modern Medicine, 1st ed., v sl. 1, p. 808. Philadelphia, Lea.

Gertler, M. M., Garn, S. M., and Levine, S. A. (1951). Annals of iniernal Medicine, 34, 1421.

Hansen, O. E. (1965). Acta Neurologica Scandinavica, 41, 357.

Henry, R. J. (1960). Clinical Chemistry: Principles and Techniques. New York, Hoeber.

Huchard, H. (1899). Traité clinique des maladies du caur et de l'aorie, 3rd ed., p. 174. Paris, Doin.

Kellgren, J. H. (1964). Annals of the Rheumatic Diseases, 23, 109.

Kohn, P. M., and Prozan, G. B. (1959). Fournal of the American Medical Association, 170, 1909.

Meyer, J. S., Kypros, G. P., and Gilroy, J. (1964). West Virginia Medical fournal, 60, 150 .

Pearce, J., and Aziz, H. (1969). Fournal of Neurology, Neurosurgery and Psychiatry. In press.

Pearson, S., Sterm, S., and Mackett, T. H. (1953). Analytical Chemistry, 25, 5 .

Schrade, W., Boehle, E., and Biegler, R. (1960). Lancet, 2, 1409.

Traut, E. F., Knight, A. A., and Szanto, P. B. (1954). fournal of the Inerican Medical Association, 156, 591.
Although the occurrence of epileptic seizures in patients taking drugs of the phenothiazine group has been known for 15 years,

- Medical Registrar, Whipps Cross Hospital, London E.11.

t Consultant Neurologist, Whipps Cross Hospital, London E.11, such attacks appear to be rare in clinical practice. When in 1959 the tricyclic drug imipramine was introduced for endogenous depression it was not at first realized that this drug was also potentially epileptogenic. Kiloh, Davison, and Osselton (1961) found that imipramine in therapeutic doses increased the frequency of attacks in epileptic patients, and that in large doses it precipitated attacks in non-epileptics, and occasionally therapeutic doses had the same effect. Amitriptyline, introduced in 1960, was found to have anticonvulsant properties in animals (Vernier, 1961), and Winfield and Aivazian (1962) reported that this drug had no effect on the electroencephalogram (E.E.G.). Davison (1965) reported that intravenous amitriptyline had an activating action on paroxysmal activity in the E.E.G.s of 11 out of 20 epileptics, and two patients who had taken overdoses of the drug developed convulsions.

Betts, Kalra, Cooper, and Jeavons (1968) reported seven cases in which it appeared that amitriptyline was the probable cause of epileptic fits in depressed patients. They emphasized that this and other antidepressant drugs should be used with caution, especially in patients whose convulsive threshold might be low, and advised that such patients should be closely supervised, 